\title{
Beta-Lactam Antibiotic Resistance among Enterobacter spp. Isolated from Infection in Animals
}

\author{
Melissa S. Wilberger ${ }^{1 *}$, Kate E. Anthony ${ }^{1 *}$, Sasha Rose ${ }^{1,2}$, Matt McClain $^{3}$, Luiz E. Bermudez ${ }^{1,2 \#}$ \\ ${ }^{1}$ Department of Biomedical Sciences, College of Veterinary Medicine, Oregon State University, Corvallis, USA \\ ${ }^{2}$ Department of Microbiology, College of Science, Oregon State University, Corvallis, USA \\ ${ }^{3}$ Information Technology, College of Veterinary Medicine, Oregon State University, Corvallis, USA \\ Email: "Luiz.Bermudez@oregonstate.edu
}

Received December 31, 2011; revised February 21, 2012; accepted March 7, 2012

\begin{abstract}
Nosocomial infections are frequent complications of hospitalization, caused by opportunistic pathogens that gain access to hosts undergoing invasive procedures, such as surgery, intubation, and placement of deep vein lines. Nosocomial infections in animal hospitals can infect other animals, as well as be transmitted to human personnel. Enterobacter is a genus of common gram-negative bacteria, which can be associated with antibiotic resistant hospital infections. Because of an outbreak in antibiotic resistance in the genus, we decided to investigate five years of Enterobacter infections in the Large Animal Services of the Lois Bates Acheson Veterinary Teaching Hospital (LBAVTH) at Oregon State University. The demographics from 37 Enterobacter-infected patients of the LBAVTH were obtained from charts and analyzed. The identified clusters of infections suggested possible patient-environment sources of infection. The environment of the hospital was sampled in an attempt to determine the source of infection. Although Enterobacter was not isolated, three of the collected samples contained bacteria with resistance to third-generation cephalosporins. Enterobacter isolates from six of the 37 patients were further analyzed for presence of specific ESBL resistance genes. All six of the isolates harbored multiple extended-spectrum beta-lactamase genes, i.e., CTX-M-15, TEM-80, SHV-2 and AmpC. In summary, Enterobacter infection in the veterinary hospital was caused by beta-lactam-resistant strains, carrying ESBL-resistant genes. Veterinary hospital personnel should be aware of the potential for transmission, to both humans and animals, of ESBL-gene-containing bacteria.
\end{abstract}

Keywords: Enterobacter spp.; Extended-Spectrum Beta-Lactamase; Cephalosporin; Epidemiology; Veterinary Medicine

\section{Introduction}

In the 1970s, Enterobacter was first noted as a common cause of nosocomial infections in immuno-compromised hosts [1-4]; with respiratory, urinary, and gastrointestinal tracts being the most common sites of infection [3]. According to the National Nosocomial Infections Surveillance System (NNIS), Enterobacter spp. accounted for $5 \%$ to $7 \%$ of hospital-acquired human infections in the United States from 1976 to 1989 [4].

Enterobacter become resistant to beta lactam antibiotics by producing an extended-spectrum beta-lactamase (ESBL) protein, which breaks the beta lactam ring of the antibiotic and inactivating it. The first ESBL isolate resistant to extended-spectrum cephalosporins, specifically SHV-beta-lactamase, was reported in 1983, in Germany [5]. In the early 1990's, ESBL-containing bacteria were

\footnotetext{
*Both authors made equal contribution to the study.

${ }^{\#}$ Corresponding author.
}

identified in the United States [6]. Over the past five years, the frequency of Enterobacter resistance to third-generation cephalosporins has increased worldwide [1].

ESBLs have been described to derive from TEM and SHV-beta-lactamase genes [7]. TEM and SHV-beta-lactamases provide resistance to broad-spectrum penicillins [4]. The beta-lactamase $\mathrm{AmpC}$ gene is responsible for resistance to cefoxitin, a second-generation cephalosporin [8]. CTX-M, another ESBL, has evolved, becoming resistant to aminopenicillins, carboxypenicillins, ureidopenicillins and narrow-spectrum cephalosporins [9]. Among all, TEM beta-lactamase ESBLs are the most prevalent form found in the United States [7].

Much of the information gained about Enterobacter resistance has been obtained in human hospitals [1-3]. Studies have been completed to help deal with the challenges that resistant Enterobacter infections cause in health care. Higher morbidity, patient costs, and extended hospital stays are common problems faced when 
resistant Enterobacter species are found in the hospital setting [2]. Significantly less information is available regarding Enterobacter antibiotic resistance in veterinary medicine; however, emergence of cephalosporin-resistant Enterobacter has been recently described in veterinary hospitals, and some of this resistance has been associated with the production of ESBL. Enterobacter infections of the urinary tract, wound sites, respiratory tract, and intravenous catheter sites were recently reported in patients admitted to small animal hospitals [10].

Infections by Enterobacter-antibiotic-resistant strains in animal hospitals can be a significant problem for animals, as well as for the human personnel. It is important that animal hospitals begin to track hospital infection by antibiotic-resistant bacteria because of the potential source of human infection.

In Oregon State University's Large Animal Services of the Lois Bates Acheson Veterinary Teaching Hospital (LBAVTH), an increase in Enterobacter infections resistant to third-generation cephalosporin (ceftiofur) was recorded from 2003 to 2010 . Among the animals, equines were the most frequent host. In this retrospective study, we initiated an investigation in the summer of 2009, to obtain further information about resistant Enterobacter infections.

\section{Materials and Methods}

\subsection{Epidemiologic Investigation}

During the period of October 2003 to March 2010, sixty cultures were positive for Enterobacter, from which thirty-seven of patients admitted in Large Animal Services of the Lois Bates Acheson Veterinary Teaching Hospital (LBAVTH) were reviewed. Microbiology laboratory reports were used to determine which patients had high resistance to Ceftiofur, a third-generation cephalosporin. The criteria used as definition of hospital infections included patients with at least a 100-h hospital stay and with a positive culture of Enterobacter obtained within the time frame of the hospital stay. Medical records were used to determine animal demographics, secondary infections/conditions, hospital locations, procedures performed, and medication administered during time of stay. Environmental samples were collected throughout the LBAVTH, from soap dispensers, technician charts, hair clippers, microwaves, prep-areas, computer keyboards, and ultrasound and radiograph machines. Sterile swabs were used to wipe surfaces and then were immediately placed into tubes containing 50\% sterile water and 50\% Luria Bertani (LB) nutrient broth. Within $1 \mathrm{~h}$ of collection, samples were streaked onto LB agar plates and incubated for $24 \mathrm{~h}$ at $37^{\circ} \mathrm{C}$. Nine distinct colonies, morphologically similar to Enterobacter, were replated to obtain pure cultures. The environmental sam- pling had no temporal connection with the peak of the outbreak, but aimed at determining the possible presence of Enterobacter in the hospital environment.

\subsection{Extended-Spectrum Beta-Lactamase}

Out of the 37 cases studied, six Enterobacter isolates had been stored in the bacteriology laboratory. They were collected from patient numbers 27, 28, 34, 35, 36, and 37 . Cultures were inoculated onto sheep blood agar plates from frozen specimens and incubated for $48 \mathrm{~h}$ at $35^{\circ} \mathrm{C}$. Using the BBL ${ }^{\text {TM }}$ Prompt $^{\text {TM }}$ Inoculation System (Becton, Dickinson and Company, Franklin Lakes, NJ), bacterial suspensions were prepared to perform disk diffusion susceptibility tests (DDST) on Mueller-Hinton plates. Cefotaxime-clavulanic, cefotaxime, ceftazidime, and ceftazidime-clavulanic disks, each at a $30 \mu \mathrm{g}$ concentration, were placed roughly $20 \mu \mathrm{m}$ apart for the DDST. Klebsiella pneumoniae, resistant to third-generation cephalosporin, and Escherichia coli were used as positive and negative controls, respectively. A positive result for the presence of an ESBL was $\mathrm{a} \geq 5 \mathrm{~mm}$ increase in the zone of diffusion diameter, in comparison with the antimicrobial agent and its clavulanic acid counterpart.

To assess antibiotic resistance of the nine isolated bacterial colonies from the hospital sampling, bacteria were re-plated onto LB agar plates containing $0.9 \mathrm{mg} / \mathrm{L}$ of ceftiofur. This concentration of ceftiofur matched the concentration of the DDST. All six isolates were tested for ESBL-resistance genes using primers described in Table 1 [11-14].

\subsection{DNA Extraction}

All bacterial DNA used in this study was extracted and purified using the DNEasy Tissue Kit (Qiagen, Valencia, CA) following the manufacturer Appendix E procedure. DNA was assessed for quality and quantity with gel electrophoresis, using $1 \%$ agarose gel stained with ethidium bromide.

\subsection{PCR Identification of ESBL Genes}

Polymerase chain reaction (PCR) was used to amplify the 16 s ribosomal RNA gene of the nine hospital isolates, as well as the specific ESBL genes in both the clinical Enterobacter samples and the ceftiofur-resistant hospital isolates. Primers used are shown in Table 1. PCR was conducted using the FideliTaq system (USB, Cleveland, $\mathrm{OH})$ with cycling as follows: 35 cycles of $96^{\circ} \mathrm{C}$ for $30 \mathrm{~s}$, $55^{\circ} \mathrm{C} / 60^{\circ} \mathrm{C}$ for $30 \mathrm{~s}$, and $68^{\circ} \mathrm{C}$ for $1.5 \mathrm{~min}$. Prior to the first cycle, a temperature of $95^{\circ} \mathrm{C}$ was held for $5 \mathrm{~min}$, and at the end of the last cycle, a temperature of $68^{\circ} \mathrm{C}$ was maintained for $4 \mathrm{~min}$. PCR products were visualized with gel electrophoresis using $1 \%$ agarose gel stained with 
Table 1. Primers used for ESBL gene PCR amplification.

\begin{tabular}{|c|c|c|c|}
\hline Primer Type & Sequences & Annealing Temp $\left({ }^{\circ} \mathrm{C}\right)$ & Reference \\
\hline \multirow{4}{*}{ TEM } & For: 5'-TCAACATTTCCGTGTCG-3' & $55 / 60$ & \multirow{4}{*}{ [14] This study } \\
\hline & Rev: 5'-CTGACAGTTACCAATGCTTA-3' & \multirow{3}{*}{$55 / 60$} & \\
\hline & For: 5'-CCTTCCTGTTTTTGCTCACC-3' & & \\
\hline & Rev: 5'-ATACGGGAGGGCTTACCATC-3' & & \\
\hline \multirow{2}{*}{ OXA-3 } & For: 5'-TTCAAGCCAAGGCACGATAG-3' & \multirow[t]{2}{*}{$55 / 60$} & \multirow{2}{*}{ [14] } \\
\hline & Rev: 5'-TTCGAGTTGACTGCCGGGTTG-3' & & \\
\hline \multirow{4}{*}{ SHV } & For: 5'-TCGGGCCGCGTAGGCATGAT-3' & $55 / 60$ & \multirow{4}{*}{ [13] This study } \\
\hline & Rev: 5'-AGCAGGGCGACAATCCCGCG-3' & & \\
\hline & For: 5'-GCCGCTTGAGCAAATTAAAC-3' & $55 / 60$ & \\
\hline & Rev: 5'-CGTATCCCGCAGATAAATCAC-3' & & \\
\hline \multirow{4}{*}{ CTX-M } & For: 5'-CGCTTTGCGATGTGCAG-3' & $55 / 60$ & \multirow{4}{*}{ [12] This study } \\
\hline & Rev: 5'-ACCGCGATATCGTTGGT-3' & & \\
\hline & For: 5'-GACGTCCGTATTTGCCTTTC-3' & $55 / 60$ & \\
\hline & Rev: 5'-TAGGTTGAGGCTGGGTGAAG-3' & & \\
\hline \multirow[t]{3}{*}{$\mathrm{AmpC}$} & For: 5'-CCCTTTGCTGCGCCCTGC-3' & $55 / 60$ & \multirow[t]{2}{*}{ [11] This study } \\
\hline & Rev: 5'-TGCCGCCTCAACGCGTGC-3' & & \\
\hline & For: 5'-CGGTGGCCGTTATTTATCAG-3' & $55 / 60$ & \multirow{4}{*}{ This study } \\
\hline \multirow{3}{*}{$16 \mathrm{~S}$} & Rev: 5'-GCCAGCGCTACCTTACTGTC-3' & & \\
\hline & For: 5'-CAGCCGCGGTAATACGTAGG-3' & 60 & \\
\hline & Rev: 5'-CGGTACGGCTACCTTGTTACG-3' & & \\
\hline
\end{tabular}

ethidium bromide. Bands of interest were cut out and extracted using the PrepEase Gel Extraction Kit (USB, Cleveland, $\mathrm{OH}$ ). Extracted samples were submitted to the Center for Genome Research and Biocomputing (CGRB) at Oregon State University for DNA sequencing. Database search and sequence comparisons were performed using the BLAST network service at the National Center for Biotechnology Information (NCBI).

\subsection{Statistical Analysis}

Statistical evaluation was performed using 2-tailed Student's $t$-test and $X^{2}$ test for comparisons between groups. A $p$ value of less than 0.5 was considered significant.

\section{Results}

\subsection{Epidemiological Results}

Sixty strains of Enterobacter were isolated from hospital infection from 2003 to 2010. Out of 60, 37 cases of infection in the LBAVTH were studied. Patients involved in each outbreak were rarely in the same stall or in close enough proximity to spread the bacteria through direct contact.

An overall view showed that most cases had Enterobacter growth from infection in urinary tract, reproduction system, bone fractures, and surgical incision sites (Table 2). However, there was no age or gender correlation found in patients with Enterobacter infections. Some cases had multiple hospital stays. Table 3 shows the percentage of resistance to third-generation cephalosporins, gentamicin and enrofloxacin (quinolone). Resistance to third-generation cephalosporin increased from 16\% among Enterobacter isolates (2002) to $71.4 \%$ in $2003,64 \%$ in $2004,78.5 \%$ in $2005,80 \%$ in $2006,88.8 \%$ in $2007,80 \%$ in $2008,42 \%$ in 2009 and $18 \%$ in 2010 (Table 3). Males accounted for $56.8 \%$ of total patients, and females accounted for $43.2 \%$ of total patients recorded. The average age was 10 years old. Observations from the patients' records in comparison to their Enterobacter susceptibility (Table 4) indicated that infection with Enterobacter strains was found through the hospital in multiple waves of outbreaks.

No Enterobacter was found in the environmental samples. However, we decided to investigate if ESBL genes were common in the bacteria isolated. From the 39 samples collected in the environmental survey (collection was performed when the peak of the resistance was over), the nine selected colonies were identified as: Micrococcus (2), Gordonia, Exiguobacterium, Dietzia, Bacillus pumilus, Bacillus spp., Paenibacillus, found on surgical hair trimmers, betadine container in the minor surgical suite, senior student room copy machine, technician chart in the hallway, and the radiology room computer, respectively (Table 5). Of the nine isolates, strains of Bacillus spp., Bacillus pumilus, and Gordonia showed resistance on the ceftiofur-inoculated plates. However, the ESBL primers amplified products corresponding to ESBL genes in none of the three resistant isolates.

\subsection{Extended-Spectrum Beta-Lactamase}

To determine whether ESBL was associated with the beta-lactamase resistance of six isolates obtained from 
Table 2. Relevant clinical aspects of the 37 patients studied with Enterobacter infection.

\begin{tabular}{|c|c|c|c|c|}
\hline & No. of participants & Percent (\%) & Average & $p$ value \\
\hline \multicolumn{5}{|l|}{ Animal Species } \\
\hline Equine & 33 & 89.2 & & $\mathrm{p}>0.05$ \\
\hline Camelid & 3 & 8.1 & & $\mathrm{p}>0.05$ \\
\hline Porcine & 1 & 2.7 & & $\mathrm{p}>0.05$ \\
\hline \multicolumn{5}{|l|}{$\underline{\operatorname{Sex}}$} \\
\hline Male & 11 & 29.7 & & $\mathrm{p}>0.05$ \\
\hline Female & 16 & 43.2 & & $\mathrm{p}>0.05$ \\
\hline Altered (neutered) & 10 & 27.0 & & $\mathrm{p}>0.05$ \\
\hline$\underline{\text { Age }}$ & & & 10 years & \\
\hline Duration of Hospitalization & & & 21 days & \\
\hline \multicolumn{5}{|l|}{$\underline{\text { Initial Diagnosis }}$} \\
\hline Colic & 13 & 35.1 & & $\mathrm{p}>0.05$ \\
\hline Limb disorders & 7 & 18.9 & & \\
\hline Reproduction & 4 & 10.8 & & \\
\hline Urinary & 3 & 8.1 & & \\
\hline Bone fractures & 5 & 13.5 & & \\
\hline Other & 5 & 13.5 & & \\
\hline
\end{tabular}

Table 3. Enterobacter infection (60 isolates) for 2003 to 2010 and the percentage of resistance of the isolates to ceftiofur, gentamicin and enrofloxacin.

\begin{tabular}{cccc}
\hline \multirow{2}{*}{ Year } & \multicolumn{3}{c}{ \% of isolates resistant to } \\
\cline { 2 - 4 } & Ceftiofur & Gentamicin & Enrofloxacin \\
\hline 2003 & 71.4 & 71.4 & 57.1 \\
2004 & 64 & 72.7 & 45.4 \\
2005 & 78.5 & 78.5 & 27.2 \\
2006 & 80 & 81.7 & 21.4 \\
2007 & 88.8 & 77.7 & 55.5 \\
2008 & 80 & 80 & 78 \\
2009 & 42 & 76 & 68 \\
2010 & 18 & 61 & 54 \\
\hline
\end{tabular}

the outbreak period, the DDST was performed and resulted in five positives (83\%) and one negative (17\%) for the presence of ESBLs (Table 6 and Figure 1). Further investigation using ESBL-specific primers to test for ESBL genes was also carried out (Table 7). None of the six clinical isolates contained exactly the same gene groups, although all contained TEM and SHV ESBL genes. Four isolates contained the AmpC ESBL gene, while only one had the CTX-M-15 ESBL gene.

In testing for similar strains using double enzyme digestion with $\mathrm{XbaI}$ and HindIII restriction enzymes, it appears that patients 34 and 35 have similar strains. Isolates from patients $27,28,36$ and 37 clearly do not share the same genetic make-up.

\section{Discussion}

Hospital infections are usually associated with resistance to third-generation cephalosporins. Members of Enterobacteriaceae produce ESBL. These organisms are an important cause of nosocomial infections, for which there are limited therapeutic options. Previous studies have established that risk factors for infections by ESBL-producing bacteria are prolonged hospital stay, previous exposure to antibiotics, urinary or vascular catheterization, intubation and mechanical ventilation and severity of disease [15].

In human medicine, Enterobacter is more frequently isolated from males and the neonatal and geriatric age ranges [4]. From information obtained in our study, no conclusion can be drawn in regard to an age range or gender that is most commonly seen in large animal patients; however, we observed that equines were the more 
Table 4. Antibiotic susceptibilities of Enterobacter isolates from 37 clinical patients studied. The table also contains information about the animal species and site of infection.

\begin{tabular}{|c|c|c|c|c|c|c|c|c|c|c|c|c|c|c|c|}
\hline Patient \# & Date & Source & Species & Ami & Amp & Ceft & Enro & Ery & $\mathbf{G}$ & $\mathbf{P}$ & Rif & Sul & Tetr & TCA & TMS \\
\hline 1 & $10 / 03$ & Muscle & Equine & $\mathrm{S}$ & $\mathrm{R}$ & $\mathrm{R}$ & I & $\mathrm{R}$ & $\mathrm{R}$ & $\mathrm{R}$ & $\mathrm{R}$ & $\mathrm{R}$ & $\mathrm{R}$ & $\mathrm{R}$ & $\mathrm{R}$ \\
\hline 2 & $10 / 03$ & Catheter/incision & Equine & S & $\mathrm{R}$ & $\mathrm{R}$ & I & $\mathrm{R}$ & $\mathrm{R}$ & $\mathrm{R}$ & $\mathrm{R}$ & $\mathrm{R}$ & $\mathrm{R}$ & $\mathrm{R}$ & $\mathrm{R}$ \\
\hline 3 & $11 / 03$ & Abdominal Fluid & Equine & $\mathrm{R}$ & $\mathrm{R}$ & S & $\mathrm{R}$ & $\mathrm{R}$ & $\mathrm{R}$ & $\mathrm{R}$ & $\mathrm{R}$ & $\mathrm{R}$ & $\mathrm{R}$ & S & $\mathrm{R}$ \\
\hline 4 & $02 / 04$ & Incision Swab & Equine & $\mathrm{S}$ & $\mathrm{R}$ & $\mathrm{R}$ & $\mathrm{R}$ & $\mathrm{R}$ & $\mathrm{R}$ & $\mathrm{R}$ & $\mathrm{R}$ & $\mathrm{R}$ & $\mathrm{R}$ & $\mathrm{R}$ & $\mathrm{R}$ \\
\hline 5 & $06 / 04$ & Catheter & Equine & S & $\mathrm{R}$ & S & $\mathrm{R}$ & $\mathrm{R}$ & $\mathrm{R}$ & $\mathrm{R}$ & $\mathrm{R}$ & $\mathrm{R}$ & $\mathrm{R}$ & S & $\mathrm{R}$ \\
\hline 6 & 07/04 & Catheter & Equine & I & $\mathrm{R}$ & I & S & $\mathrm{R}$ & $\mathrm{R}$ & $\mathrm{R}$ & $\mathrm{R}$ & $\mathrm{R}$ & $\mathrm{R}$ & I & I \\
\hline 7 & $07 / 04$ & Tendon & Equine & I & $\mathrm{R}$ & I & $\mathrm{S}$ & $\mathrm{R}$ & $\mathrm{R}$ & $\mathrm{R}$ & $\mathrm{R}$ & $\mathrm{R}$ & $\mathrm{R}$ & I & I \\
\hline 8 & $08 / 04$ & Swab & Equine & S & $\mathrm{R}$ & S & S & $\mathrm{R}$ & $\mathrm{R}$ & $\mathrm{R}$ & $\mathrm{R}$ & $\mathrm{R}$ & $\mathrm{R}$ & $\mathrm{R}$ & $\mathrm{S}$ \\
\hline 9 & $12 / 04$ & Catheter & Equine & I & $\mathrm{R}$ & I & I & $\mathrm{R}$ & $\mathrm{R}$ & $\mathrm{R}$ & $\mathrm{R}$ & $\mathrm{R}$ & $\mathrm{R}$ & I & $\mathrm{R}$ \\
\hline 9 & $12 / 04$ & Incision Swab & Equine & I & $\mathrm{R}$ & I & I & $\mathrm{R}$ & $\mathrm{R}$ & $\mathrm{R}$ & $\mathrm{R}$ & $\mathrm{R}$ & $\mathrm{R}$ & I & $\mathrm{R}$ \\
\hline 10 & $12 / 04$ & Wound Swab & Equine & I & $\mathrm{R}$ & I & I & $\mathrm{R}$ & $\mathrm{R}$ & $\mathrm{R}$ & $\mathrm{R}$ & $\mathrm{R}$ & $\mathrm{R}$ & I & $\mathrm{R}$ \\
\hline 9 & $12 / 04$ & Wound Swab & Equine & I & $\mathrm{R}$ & I & I & $\mathrm{R}$ & $\mathrm{R}$ & $\mathrm{R}$ & $\mathrm{R}$ & $\mathrm{R}$ & $\mathrm{R}$ & I & $\mathrm{R}$ \\
\hline 11 & $01 / 05$ & Catheter Site Swab & Equine & S & $\mathrm{R}$ & $\mathrm{R}$ & S & $\mathrm{R}$ & $\mathrm{R}$ & $\mathrm{R}$ & $\mathrm{R}$ & $\mathrm{R}$ & I & I & $\mathrm{R}$ \\
\hline 12 & $01 / 05$ & Catheter Site Swab & Equine & $\mathrm{S}$ & $\mathrm{R}$ & $\mathrm{R}$ & S & $\mathrm{R}$ & $\mathrm{R}$ & $\mathrm{R}$ & $\mathrm{R}$ & $\mathrm{R}$ & I & I & $\mathrm{R}$ \\
\hline 13 & $10 / 05$ & Abdominal Fluid & Equine & I & $\mathrm{R}$ & I & S & $\mathrm{R}$ & I & $\mathrm{R}$ & $\mathrm{R}$ & I & I & I & I \\
\hline 14 & $10 / 05$ & Catheter Site Swab & Equine & I & $\mathrm{R}$ & I & S & $\mathrm{R}$ & I & $\mathrm{R}$ & $\mathrm{R}$ & I & I & I & I \\
\hline 15 & $10 / 05$ & Incision Swab & Equine & I & $\mathrm{R}$ & I & S & $\mathrm{R}$ & I & $\mathrm{R}$ & $\mathrm{R}$ & I & I & I & I \\
\hline 16 & $10 / 05$ & Wound Swab & Equine & I & $\mathrm{R}$ & I & S & $\mathrm{R}$ & I & $\mathrm{R}$ & $\mathrm{R}$ & I & I & I & I \\
\hline 16 & $11 / 05$ & Abscess Swab & Equine & I & $\mathrm{S}$ & I & S & $\mathrm{R}$ & $\mathrm{R}$ & $\mathrm{R}$ & $\mathrm{R}$ & $\mathrm{R}$ & I & I & $\mathrm{R}$ \\
\hline 17 & $11 / 05$ & Incision Swab & Equine & I & $\mathrm{S}$ & I & S & $\mathrm{R}$ & $\mathrm{R}$ & $\mathrm{R}$ & $\mathrm{R}$ & $\mathrm{R}$ & I & I & $\mathrm{R}$ \\
\hline 18 & $11 / 05$ & Abscess Swab & Camelid & I & $\mathrm{R}$ & I & S & $\mathrm{R}$ & - & $\mathrm{R}$ & - & $\mathrm{R}$ & I & - & $\mathrm{R}$ \\
\hline 19 & $11 / 05$ & Uterine Biopsy & Camelid & I & $\mathrm{R}$ & I & S & $\mathrm{R}$ & $\mathrm{R}$ & $\mathrm{R}$ & $\mathrm{R}$ & $\mathrm{R}$ & I & S & $\mathrm{R}$ \\
\hline 20 & $01 / 06$ & Urine & Equine & S & $\mathrm{R}$ & $\mathrm{R}$ & S & $\mathrm{R}$ & $\mathrm{R}$ & $\mathrm{R}$ & $\mathrm{R}$ & $\mathrm{R}$ & S & $\mathrm{R}$ & $\mathrm{R}$ \\
\hline 21 & 03/06 & Bone & Equine & S & S & S & S & $\mathrm{R}$ & S & $\mathrm{R}$ & $\mathrm{R}$ & $\mathrm{R}$ & $\mathrm{R}$ & S & $\mathrm{R}$ \\
\hline 22 & 03/06 & Urine & Porcine & S & $\mathrm{R}$ & $\mathrm{R}$ & $\mathrm{R}$ & - & - & $\mathrm{R}$ & - & $\mathrm{R}$ & $\mathrm{R}$ & - & $\mathrm{R}$ \\
\hline 23 & $05 / 06$ & Blood Culture & Equine & I & $\mathrm{R}$ & I & I & $\mathrm{R}$ & I & $\mathrm{R}$ & $\mathrm{R}$ & I & I & I & I \\
\hline 24 & $05 / 06$ & Catheter Site Swab & Equine & I & $\mathrm{R}$ & I & I & $\mathrm{R}$ & I & $\mathrm{R}$ & $\mathrm{R}$ & I & I & I & I \\
\hline 24 & $05 / 06$ & Catheter & Equine & I & $\mathrm{R}$ & I & I & $\mathrm{R}$ & I & $\mathrm{R}$ & $\mathrm{R}$ & I & I & I & I \\
\hline 25 & $06 / 06$ & Catheter & Equine & I & $\mathrm{R}$ & I & I & $\mathrm{R}$ & I & $\mathrm{R}$ & $\mathrm{R}$ & I & I & S & I \\
\hline 26 & $06 / 06$ & Peritoneal Fluid & Equine & I & $\mathrm{R}$ & I & I & $\mathrm{R}$ & I & $\mathrm{R}$ & $\mathrm{R}$ & I & I & S & I \\
\hline 27 & $07 / 06$ & Catheter Site Swab & Equine & I & $\mathrm{R}$ & I & $\mathrm{S}$ & $\mathrm{R}$ & $\mathrm{R}$ & $\mathrm{R}$ & $\mathrm{R}$ & $\mathrm{R}$ & $\mathrm{R}$ & $\mathrm{R}$ & $\mathrm{R}$ \\
\hline 28 & 07/06 & Incision Swab & Equine & I & $\mathrm{R}$ & I & $\mathrm{S}$ & $\mathrm{R}$ & $\mathrm{R}$ & $\mathrm{R}$ & $\mathrm{R}$ & $\mathrm{R}$ & $\mathrm{R}$ & $\mathrm{R}$ & $\mathrm{R}$ \\
\hline 29 & $04 / 07$ & Catheter Site Swab & Equine & S & $\mathrm{R}$ & $\mathrm{R}$ & S & $\mathrm{R}$ & $\mathrm{R}$ & $\mathrm{R}$ & $\mathrm{R}$ & $\mathrm{R}$ & $\mathrm{R}$ & S & $\mathrm{R}$ \\
\hline 30 & $05 / 07$ & Urine & Equine & $\mathrm{S}$ & $\mathrm{R}$ & $\mathrm{R}$ & $\mathrm{R}$ & $\mathrm{R}$ & $\mathrm{R}$ & $\mathrm{R}$ & $\mathrm{R}$ & $\mathrm{R}$ & $\mathrm{R}$ & I & $\mathrm{R}$ \\
\hline 31 & 08/07 & Incision Swab & Equine & S & $\mathrm{R}$ & $\mathrm{R}$ & $\mathrm{R}$ & $\mathrm{R}$ & $\mathrm{R}$ & $\mathrm{R}$ & $\mathrm{R}$ & $\mathrm{R}$ & $\mathrm{R}$ & $\mathrm{R}$ & $\mathrm{R}$ \\
\hline 32 & $09 / 07$ & Tissue & Equine & S & $\mathrm{R}$ & $\mathrm{R}$ & $\mathrm{R}$ & $\mathrm{R}$ & $\mathrm{R}$ & $\mathrm{R}$ & $\mathrm{R}$ & $\mathrm{R}$ & $\mathrm{R}$ & $\mathrm{S}$ & $\mathrm{R}$ \\
\hline 32 & $10 / 07$ & Tissue & Equine & I & $\mathrm{R}$ & $\mathrm{R}$ & $\mathrm{R}$ & $\mathrm{R}$ & $\mathrm{R}$ & $\mathrm{R}$ & $\mathrm{R}$ & $\mathrm{R}$ & $\mathrm{R}$ & $\mathrm{R}$ & $\mathrm{R}$ \\
\hline 33 & $10 / 07$ & Uterine Swab & Equine & I & $\mathrm{R}$ & $\mathrm{R}$ & $\mathrm{R}$ & $\mathrm{R}$ & $\mathrm{R}$ & $\mathrm{R}$ & $\mathrm{R}$ & $\mathrm{R}$ & $\mathrm{R}$ & $\mathrm{R}$ & $\mathrm{R}$ \\
\hline 34 & $05 / 08$ & Tissue & Equine & $\mathrm{R}$ & $\mathrm{R}$ & $\mathrm{R}$ & $\mathrm{R}$ & $\mathrm{R}$ & $\mathrm{R}$ & $\mathrm{R}$ & $\mathrm{R}$ & $\mathrm{R}$ & $\mathrm{R}$ & $\mathrm{S}$ & $\mathrm{R}$ \\
\hline 35 & $10 / 08$ & Catheter & Equine & $\mathrm{R}$ & $\mathrm{R}$ & $\mathrm{R}$ & $\mathrm{R}$ & $\mathrm{R}$ & $\mathrm{R}$ & $\mathrm{R}$ & $\mathrm{R}$ & $\mathrm{R}$ & $\mathrm{R}$ & $\mathrm{R}$ & $\mathrm{R}$ \\
\hline 36 & $12 / 08$ & Swab & Equine & $\mathrm{S}$ & I & $\mathrm{R}$ & I & $\mathrm{R}$ & I & $\mathrm{R}$ & $\mathrm{R}$ & I & $\mathrm{R}$ & $\mathrm{R}$ & I \\
\hline 37 & 03/09 & Urine & Camelid & $\mathrm{S}$ & $\mathrm{R}$ & $\mathrm{R}$ & $\mathrm{R}$ & $\mathrm{R}$ & $\mathrm{R}$ & $\mathrm{R}$ & $\mathrm{R}$ & $\mathrm{R}$ & $\mathrm{R}$ & $\mathrm{R}$ & $\mathrm{S}$ \\
\hline
\end{tabular}

Ami: amikacin; Amp: ampicillin; Ceft: ceftiofur; Enro: enrofloxacin; Ery: erythromycin; G: gentamicin; P: penicillin; Rif: rifampin; Sul: sulfisoxazole; Tetr: tetracycline; TCA: Ticarcillin-clavulanic acid; TMS: trimethoprim sulfamethoxazole; R: resistant; S: susceptible; I: intermediate. 
Table 5. Location of hospital environmental samples and identification of correspondent isolates.

\begin{tabular}{cc}
\hline Hospital location & Organism(s) isolated \\
\hline Minor surgical suite & \\
Hair trimmers & Bacillus $^{*}$, Exiguobacterium $^{*}$ \\
Betadine container & Gordonia $^{*}$ \\
Senior student room, copy machine & Dietzia, Micrococcus \\
Technician cart & \\
Hallway & Bacillus pumilus $^{*}$ \\
Near stall E-14 & Paenibacillus \\
Radiology suite (Computer) & Bacillus spp. \\
\hline
\end{tabular}

*Organisms resistant to ceftiofur (third-generation cephalosporin).

Table 6. ESBL-positive results (Disk Diffusion Test) for 6 clinical isolates of Enterobacter.

\begin{tabular}{|c|c|c|c|}
\hline Name & Drug & $\mathbf{m m}$ & $\operatorname{MIC}(\mu \mathrm{g} / \mathrm{ml})$ \\
\hline \multirow[t]{4}{*}{ Patient No. $27^{*}$} & Cefotaxime-clavulanic & 18 & \\
\hline & Cefotaxime & 18 & 12 \\
\hline & Ceftazidime & 15 & 32 \\
\hline & Ceftazidime-clavulanic & 27 & \\
\hline \multirow[t]{4}{*}{ Patient No. $34^{*}$} & Cefotaxime-clavulanic & 23 & \\
\hline & Cefotaxime & 15 & 24 \\
\hline & Ceftazidime & 10 & 96 \\
\hline & Ceftazidime-clavulanic & 23 & \\
\hline \multirow[t]{4}{*}{ Patient No. $35^{*}$} & Cefotaxime-clavulanic & 24 & \\
\hline & Cefotaxime & 19 & 10 \\
\hline & Ceftazidime & 13 & 48 \\
\hline & Ceftazidime-clavulanic & 26 & \\
\hline \multirow[t]{4}{*}{ Patient No. $28^{*}$} & Cefotaxime-clavulanic & 29 & \\
\hline & Cefotaxime & 20 & 8 \\
\hline & Ceftazidime & 16 & 24 \\
\hline & Ceftazidime-clavulanic & 27 & \\
\hline \multirow[t]{4}{*}{ Patient No. $36^{*}$} & Cefotaxime-clavulanic & 19 & \\
\hline & Cefotaxime & 21 & 6 \\
\hline & Ceftazidime & 13 & 48 \\
\hline & Ceftazidime-clavulanic & 24 & \\
\hline \multirow[t]{4}{*}{ Patient No. 37} & Cefotaxime-clavulanic & 10 & \\
\hline & Cefotaxime & 12 & 64 \\
\hline & Ceftazidime & 9 & 128 \\
\hline & Ceftazidime-clavulanic & 10 & \\
\hline
\end{tabular}

*ESBL positive determined by a $\geq 5 \mathrm{~mm}$ diameter difference between antimicrobial agent and clavulanic counterpart.

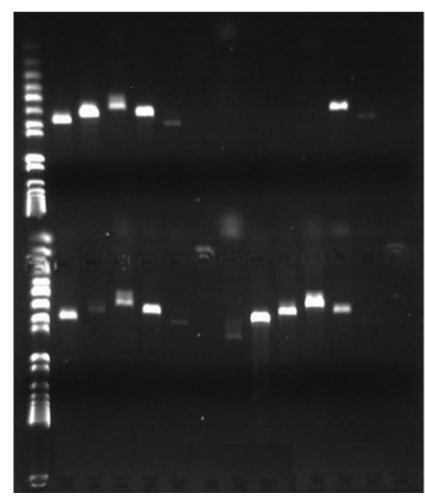

(a)

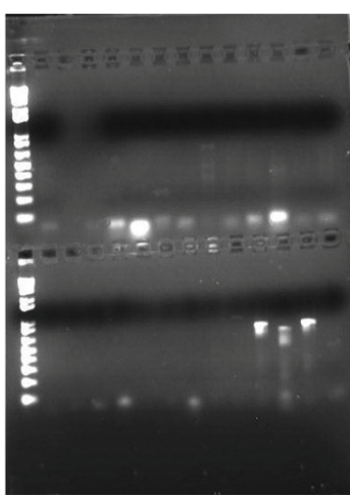

(b)

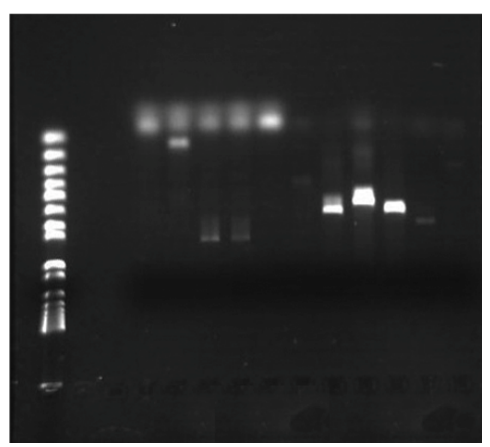

(c)

Figure 1. (a) ESBL primers electrophoresis check on $1 \%$ agarose gel stained with ethidium bromide of six clinical isolates and three collected samples from LBAVTH. Top row: DNA marker, patient 36 AmpC, SHV, TEM, TEM, CTXM; patient 37 AmpC, AmpC, SHV, SHV, TEM, TEM, CTXM. Bottom row: DNA marker, patient 35 AmpC, SHV, SHV, TEM, TEM, CTXM; patient 34 AmpC, AmpC, SHV, SHV, TEM, TEM, CTXM; (b) ESBL primers electrophoresis check on $1 \%$ agarose gel stained with ethidium bromide of six clinical isolates and three collected samples from LBAVTH. Top row: DNA marker, Bacillus sp. CTXM, TEM, TEM, SHV, AmpC, AmpC, SHV, Gordonia CTXM, TEM, TEM, SHV, SHV, AmpC. Bottom row: DNA marker, Bacillus pumilus, TEM, CTXM, TEM, SHV, SHV, AmpC, AmpC, patient 28 CTXM, TEM, CTXM, SHV, SHV, AmpC; (c) ESBL primers electrophoresis check on 1\% agarose gel stained with ethidium bromide of six clinical isolates and three collected samples from LBAVTH. Row: DNA marker, patient 28 AmpC, Gordonia AmpC, patient 36 AmpC, patient 36 AmpC, patient 27 AmpC, AmpC, SHV, SHV, TEM, TEM, CTXM.

common host for Enterobacter in comparison to porcine and camelids. Equine patients, moreover, were admitted much more frequently into the LBAVTH than other species, making any conclusion regarding species-related predisposition not possible.

Enrofloxacin, a commonly used quinolone, had an overall increase in resistance, especially in 2007 and 2008. Gentamicin was an antibiotic commonly prescribed in the hospital, and the usage is probably reflected in an increased resistance of Enterobacter strains to $88.8 \%$. 
Table 7. Identification of ESBL genes by Real-Time PCR amplification.

\begin{tabular}{ccccccc}
\hline Gene & Patient 27 & Patient 28 & Patient 34 & Patient 35 & Patient 36 & Patient 37 (-) \\
\hline CTX-M-15 & no & no & no & No & yes & no \\
TEM 80 & yes & yes & yes & Yes & yes & yes \\
SHV 2 & yes & yes & yes & Yes & yes & yes \\
AmpC & no & yes & yes & Yes & yes & no \\
\hline
\end{tabular}

$(-)$ patient tested negative, with Disk Diffusion Test, for presence of ESBL.

It seems that, in some of the patients examined, such as patients $6-10$, the infection was nosocomial. While the patients from this group, in general, had positive Enterobacter cultures from catheters or incision sites after surgery, patient 10, 2 days after admittance, was cultured positive for Enterobacter from a pre-existing heel bulb laceration infection. Patient 9 entered the hospital after patient 10 was diagnosed with an Enterobacter infection and stayed in the same stall that patient 10 previously occupied. Patient 9 cultured positive for Enterobacter that had a complete susceptibility match to patient 10's culture. Although this does not represent definitive evidence that the same bacterial strain infected both horses, it is worthy of notice.

A wave of patients with infection caused by organisms with similar susceptibilities was also seen between patients 13 - 16. This set of patients did not stall near each other. The only similarity in procedures is that all had ultrasounds. However, there are multiple ultrasound machines within the LBAVTH, making it impossible to conclude which machine was used and the source of transmission. Interestingly, patient 16 cultured for two different strains of Enterobacter, where the second cultured strain was more resistant and similar to the susceptibility pattern of patients 17 - 19's culture.

Another set of infections by bacteria with similar susceptibilities was seen in patients $23-26$. Here, it is clear that patient 23 was an index case. A premature day-old foal was admitted and its blood cultured positive for Enterobacter within the same day. This foal died by cardiac arrest. The next case, patient 24, was admitted 4 days later and cultured positive for Enterobacter 3 weeks afterwards. Both underwent radiographs, although the transmission mechanism remains inconclusive. The other two similar cases (patients 25,26 ) seem to indicate secondary nosocomial infections, which are assumed to be transmitted from the earlier patients. Beginning July 2006, no other waves of infection could be confidently noted (Table 4).

The transmission path of Enterobacter is obscure, though data would suggest that hospital personnel transmission could be a likely source of infection. During each wave of Enterobacter infection in the hospital, pa- tients were rarely in close enough contact to spread the bacteria animal-to-animal. Human medicine studies have also found that personnel transmission is a form of Enterobacter transmission $[1,3,16]$. In 2003, patients 1 and 2 overlapped by 5 days and were in neighboring stalls. In 2004, patients 7 and 8 overlapped by 22 days and were in similar areas of the hospital. Also, patients 9 and 10 overlapped by 25 days and stayed, at different times, in the same stall. In 2005, patients 14 - 19 all overlapped within a one-month period. In 2006, patients 22 and 23 overlapped by 2 days; while patients 25 and 26 overlapped by 3 days. Patient 25 also overlapped with patient 27 by 8 days. There was no significant overlap observed for the years 2007 and 2008.

Common medical procedures performed in the LBAVTH are also possible forms of transmission. This is well documented for human medicine, as well [17]. Procedures that were found to be common among the 37 patients within the LBAVTH during an Enterobacter wave included radiographs (51\%), clean surgery $(27 \%)$, dirty surgery (46\%), abdominocentesis (41\%), endoscopy $(16 \%)$ and ultrasound (51\%). It was observed that $50 \%$ of Enterobacter-positive patients underwent either radiographs or ultrasounds. With such a high percentage of use, these devices can become possible harbors for bacteria.

In the environmental survey of the LBAVTH, there was no known pathogens found. However, the survey was carried out when the peak of the outbreak was long past, and the goal of it was to determine whether Enterobacter could be found from the environment or whether any other organism found would harbor ESBL. From the samples collected, three of the nine showed resistance to ceftiofur. The known bacteria that were resistant were: Gordonia spp., Bacillus spp. and Bacillus pumilus. Gordonia is a gram-positive to gram-variable bacteria commonly found in the environment. It can be pathogenic in immuno-suppressed human hosts [18]. Its presence in the LBAVTH may be attributed to Gordonia's natural environmental habitat and presence on patients before admittance. Although no Enterobacter was found in the hospital environmental survey, its presence or absence remains inconclusive, as many surveys seem 
to be unable to find the bacteria within the hospital environment $[3,19]$. A possibility is that environmental bacteria over-grew infection-associated pathogens. A more effective survey could be performed with a vigilant watch of Enterobacter cases admitted and then executing environmental surveys during periods of increased cases.

From the six Enterobacter isolates stored, five tested positive for possible ESBL presence from the DDST. In confirmation of the DDST, the samples were further evaluated by testing for ESBL gene presence with PCR. The rational for using the PCR screening method was because DDST is not a truly effective means to determine ESBL presence [20]. The results from the PCR showed that all six clinical isolates had, at least, genes for both TEM and SHV. Patient 36 is interesting in that all the primers amplified for genes TEM, SHV, CTXM-15, and AmpC. Patient 35, who amplified all but CTX-M-15, had a greater overall resistance than patient 36. Of note, is that the plasmid carrying CTX-M-15 usually also carries resistant genes for fluoroquinolones [9, 21 ] and can explain the high resistance to enrofloxacin.

Intracellular protein profiles were also compared among Enterobacter strains. Patients 34 and 35 appear to harbor the same strain (data not shown). Interestingly, they were all cultured within a 7-mo time span. Both patients seem to have developed nosocomial infections. Since there were no stored samples from all past patients, it is difficult to determine the index case. It is important to note there were no similar procedures carried out among all three patients; they were not stalled in the same location; nor did their hospital stays overlap. This could potentially be an indication of hospital personnel transmission as personnel contact. The other four clinical isolates (patients $27,28,36,37$ ) did not carry the same strain. However, patients 27 and 28 had similar susceptibilities and were also in the hospital at the same time.

In summary, we describe an investigation on the sixyear period of increased Enterobacter resistance to cephalosporins at the LBAVTH. We found that all of the stored strains reminiscent of the period of time carried ESBL and were also resistant to fluoroquinolones. Humans coming into contact with animal patients infected with Enterobacter should be aware of the chance of carrying ESBL genes to outside of the hospital location.

\section{Acknowledgements}

We thank Kristi Crofoot, Jilleen Pfaff, Peggy Dearing, Mandy Seals, Elizabeth Harbert, Denny Weber and Becky Paasch for their help in obtaining information necessary for this study. We thank Denny Weber for help with the manuscript. Also, we thank Drs. Shannon K. Reed, Stacy Semevolos and Christopher Cebra for their contribution from various clinical cases.

\section{REFERENCES}

[1] C. Arpin, C. Coze, A. M. Rogues, J. P. Gachie, C. Bebear and C. Quentin, "Epidemiological Study of an Outbreak Due to Multidrug-Resistant Enterobacter aerogenes in a Medical Intensive Care Unit," Journal of Clinical Microbiology, Vol. 34, No. 9, 1996, pp. 2163-2169.

[2] S. E. Cosgrove, K. S. Kaye, G. M. Eliopoulous and Y. Carmeli, "Health and Economic Outcomes of the Emergence of Third-Generation Cephalosporin Resistance in Enterobacter Species," Archives of Internal Medicine, Vol. 162, No. 2, 2002, pp. 185-190. doi:10.1001/archinte.162.2.185

[3] S. Jalaluddin, J. M. Devaster, R. Scheen, M. Gerard and J. P. Butzler, "Molecular Epidemiological Study of Nosocomial Enterobacter aerogenes Isolates in a Belgian Hospital," Journal of Clinical Microbiology, Vol. 36, No. 7, 1998, pp. 1846-1852.

[4] W. E. Sanders Jr. and C. C. Sanders, "Enterobacter spp.: Pathogens Poised to Flourish at the Turn of the Century," Clinical Microbiology Reviews, Vol. 10, No. 2, 1997, pp. 220-241.

[5] P. E. Coudron, E. S. Moland and C. C. Sanders, "Occurrence and Detection of Extended-Spectrum Beta-Lactamases in Members of the Family Enterobacteriaceae at a Veterans Medical Center: Seek and You May Find," Journal of Clinical Microbiology, Vol. 35, No. 10, 1997, pp. 2593-2597.

[6] L. B. Rice, S. H. Willey, G. A. Papanicolaou, A. A. Medeiros, G. M. Eliopoulos, R. C. Moellering Jr. and G. A. Jacoby, "Outbreak of Ceftazidime Resistance Caused by Extended-Spectrum Beta-Lactamases at a Massachusetts Chronic-Care Facility," Antimicrobial Agents and Chemotherapy, Vol. 34, No. 11, 1990, pp. 2193-2199.

[7] L. A. Minarini, E. C. Clímaco, D. B. Guimarães, J. C. Ferreira, I. C. Palazzo, R. Martinez and A. L. Darini, "Clonal Transmission of ESBL-Producing Klebsiella spp. at a University Hospital in Brazil," Current Microbiology, Vol. 56, No. 6, 2008, pp. 587-591.

doi:10.1007/s00284-008-9129-5

[8] N. D. Hanson, "AmpC Beta-Lactamases: What Do We Need to Know for the Future?" Journal of Antimicrobial Consumption, Vol. 52, No. 1, 2003, pp. 2-4. doi:10.1093/jac/dkg284

[9] R. Bonnet, "Growing Group of Extended-Spectrum BetaLactamases: The CTX-M Enzymes," Antimicrobial Agents and Chemotherapy, Vol. 48, No. 1, 2004, pp. 1-14. doi:10.1128/AAC.48.1.1-14.2004

[10] J. S. Weese, "Investigation of Enterobacter cloacae Infections at a Small Animal Veterinary Teaching Hospital," Veterinary Microbiology, Vol. 130, No. 3-4, 2008, pp. 426-428. doi:10.1016/j.vetmic.2008.02.009

[11] L. Bret, C. Chanal-Claris, D. Sirot, E. B. Chaibi, R. Labia and J. Sirot, "Chromosomally Encoded AmpC-Type BetaLactamase in a Clinical Isolate of Proteus mirabilis," Antimicrobial Agents and Chemotherapy, Vol. 42, No. 5, 1998, pp. 1110-1114.

[12] C. Dutour, R. Bonnet, H. Marchandin, M. Boyer, C. Chanal, D. Sirot and J. Sirot, "CTX-M-1, CTX-M-3, and 
CTX-M-14 Beta-Lactamases from Enterobacteriaceae Isolated in France," Antimicrobial Agents and Chemotherapy, Vol. 46, No. 2, 2002, pp. 534-537. doi:10.1128/AAC.46.2.534-537.2002

[13] M. Quinteros, M. Radice, N. Gardella, M. M. Rodriguez, N. Costa, D. Korbenfeld, E. Couto and G. Gutkind, "Extended-Spectrum Beta-Lactamases in Enterobacteriaceae in Buenos Aires, Argentina, Public Hospitals," Antimicrobial Agents and Chemotherapy, Vol. 47, No. 9, 2003, pp. 2864-2867. doi:10.1128/AAC.47.9.2864-2867.2003

[14] J. Schlesinger, S. Navon-Venezia, I. Chmelnitsky, O. Hammer-Munz, A. Leavitt, H. S. Gold, M. J. Schwaber and Y. Carmeli, "Extended-Spectrum Beta-Lactamases among Enterobacter Isolates Obtained in Tel Aviv, Israel," Antimicrobial Agents and Chemotherapy, Vol. 49, No. 3, 2005, pp. 1150-1156. doi:10.1128/AAC.49.3.1150-1156.2005

[15] J. D. Pitout, D. B. Gregson, D. L. Church, S. Elsayed and K. B. Laupland, "Community-Wide Outbreaks of Clonally Related CTX-M-14 Beta-Lactamase-Producing Escherichia coli Strains in the Calgary Health Region," Journal of Clinical Microbiology, Vol. 43, No. 6, 2005, pp. 28442849. doi:10.1128/JCM.43.6.2844-2849.2005

[16] X. Bertrand, D. Hocquet, K. Boisson, E. Siebor and P. Plesiat, D. Talon, "Molecular Epidemiology of Enterobacteriaceae Producing Extended-Spectrum Beta-Lactamase in a French University-Affiliated Hospital," The International Journal of Antimicrobial Agents, Vol. 22, No. 2, 2003, pp. 128-133.
doi:10.1016/S0924-8579(03)00098-0

[17] C. Neuwirth, E. Siebor, J. Lopez, A. Pechinot and A. Kazmierczak, "Outbreak of TEM-24-Producing Enterobacter aerogenes in an Intensive Care Unit and Dissemination of the Extended-Spectrum Beta-Lactamase to Other Members of the Family Enterobacteriaceae," Journal of Clinical Microbiology, Vol. 34, No. 1, 1996, pp. 76-79.

[18] O. Lesens, Y. Hansmann, P. Riegel, R. Heller, M. Benaissa-Djellouli, M. Martinot, H. Petit and D. Christmann, "Bacteremia and Endocarditis Caused by a Gordonia Species in a Patient with a Central Venous Catheter," Emerging Infectious Diseases, Vol. 6, No. 4, 2000, pp. 382-385. doi:10.3201/eid0604.000410

[19] J. Duchon, P. Graham III, P. Della-Latta, S. Whittier, D. Carp, D. Bateman and L. Saiman, "Epidemiology of Enterococci in a Neonatal Intensive Care Unit," Infection Control \& Hospital Epidemiology, Vol. 29, No. 4, 2008, pp. 374-376. doi:10.1086/533544

[20] E. Tzelepi, P. Giakkoupi, D. Sofianou, V. Loukova, A. Kemeroglou and A. Tsakris, "Detection of Extended-Spectrum Beta-Lactamases in Clinical Isolates of Enterobacter cloacae and Enterobacter aerogenes," Journal of Clinical Microbiology, Vol. 38, No. 2, 2000, pp. 542-546.

[21] A. Y. Peleg and D. C. Hooper, "Hospital-Acquired Infections Due to Gram-Negative Bacteria," The New England Journal of Medicine, Vol. 362, No. 19, 2010, pp. 18041813. doi:10.1056/NEJMra0904124 